\title{
Local and Systemic Immune Responses to Salmonella in Genetically Susceptible I/St Mice after Mucosal Challenge
}

\author{
N. V. Kobets, L. N. Nesterenko, and D. V. Balunets \\ Gamaleya Research Institute for Epidemiology and Microbiology, Russian Ministry of Health, Gamaleya Street 18, \\ Moscow 123098, Russia \\ Correspondence should be addressed to N. V. Kobets; nkobets2000@yahoo.com
}

Received 29 March 2013; Accepted 8 May 2013

Academic Editors: A. Boon and P. Puccetti

Copyright (C) 2013 N. V. Kobets et al. This is an open access article distributed under the Creative Commons Attribution License, which permits unrestricted use, distribution, and reproduction in any medium, provided the original work is properly cited.

It was previously demonstrated that mice of I/StSnEgYCit (I/St) strain are more susceptible to tuberculosis infection than A/SnYCit (A/Sn) mice, and this susceptibility was controlled by a few interacting QTLs mapped to chromosomes 3, 9, 17. It was also shown, that I/St mice displayed higher susceptibility to acute salmonella disease after intraperitoneal challenge with $S$. enterica serovar Typhimurium. Genetic mapping showed the involvement of Tbs2 (D9Mit89) loci in control of both salmonella and tuberculosis infections. In this study we define the immunological correlates of susceptibility in I/St mice after oral administration of $S$. enterica serovar Typhimurium. We found that resistance/susceptibility in I/St and A/Sn mice in our experiments correlated with differential pattern of early local and systemic responses of innate cells and specifically with higher involvement of Gr-1 ${ }^{+}$cells in local responses of resistant mice. This correlated with higher mucosal antibody production in A/Sn mice compared to I/St. I/St mice had predominant local involvement of CD11c ${ }^{+}$cells and lack of mucosal antibodies. CD11c ${ }^{+}$cells were the major type of cells that facilitated dissemination of salmonella to the target organs, while $\mathrm{Gr}-1^{+}$cells contributed to exaggerated systemic inflammatory responses later in the course of infection. Our observations regarding the role of different cell populations in local and systemic immunity in susceptible and resistant mice are of importance for understanding of salmonella-induced cellular pathology and development of the strategy of its control.

\section{Introduction}

Salmonella are intracellular gram-negative bacteria found ubiquitously in nature and still representing a major public health problem [1]. Over 16 million cases of salmonella are associated with enteric fever, and 600000 deaths from untreated infection are reported annually in developing countries worldwide [1]. An increased incidence of nontyphoid salmonella infections with most cases tracing back to food contamination is experienced in industrialized countries [1]. The risks and outcome of salmonella infection are influenced by many factors such as antimicrobials, integrity of the host endogenous flora, loss of the gastric acid barrier, HIV infection, and host genetics [1]. Some of mouse salmonella resistance genes such as Ity, Lps, and xid were widely studied in the past decades [2-4]. Some new loci, such as Tbc2 (D9Mit89), were identified recently [5]. However, the links between susceptible host phenotype and newly identified locus were not studied in detail.
Salmonella is an enteric pathogen, and the control of bacterial colonization at mucosal surfaces depends on rapid activation of the local innate and adaptive immunity and recruitment of a wide range of cells to the site of infection [6]. Besides, the genetically mediated control of pathogen dissemination from mucosal surfaces to systemic organs is a key point for antibacterial protection. Complex interplay of different cellular subsets (neutrophils, dendritic cells, monocytes, B and T cells) at mucosal sites may lead to either transfer of bacteria to systemic lymphoid organs $[7,8]$ or preventing them from leaving mucosal organized tissue (MALT) [9]. On the other hand some factors of bacterial virulence, such as products of Spi-1 and Spi-2 loci, contribute differentially to infectious process at systemic and mucosal sites of immune system [10].

Mucosal IgA antibodies are the most abundant type of antibodies in mice and are the first to encounter mucosal pathogens even before their entry into mucosal tissue and as such are being extensively studied [11, 12]. However, 
the differences in the local cellular mechanisms mediating the control of infection in resistant mice compared to susceptible and the possible role of local defenses in the outcome of infection have not been fully assessed.

Recently it was shown that I/St and A/Sn mice opposite in their resistance to M. tuberculosis [13] display the same pattern of resistance to i.p. infection with S. enterica, serovar Typhimurium [14].

In this study we developed a model of mucosal salmonella infection in I/St and $\mathrm{A} / \mathrm{Sn}$ mice and analyzed the straindependent susceptibility to oral salmonella challenge and early innate and specific B-cell responses at mucosal and systemic lymphoid sites of both mouse strains.

As for systemic infection we showed that these strains are opposite in their resistance after oral infectious challenge with salmonella. We also showed that these strains of mice had different cellular patterns of early local innate response with preferentially $\mathrm{CD}_{11}{ }^{+}$cells involvement in I/St mice and preferentially $\mathrm{Gr}-1^{+}$cells involvement in $\mathrm{A} / \mathrm{Sn}$ mice that probably resulted in differential type of early acquired immune response and specifically early local IgA production observed only in A/Sn mice. Additionally, we showed that high bacterial load and splenomegaly observed in susceptible mice at day 7 after oral infection correlated with higher level of bright Gr-1 ${ }^{+}$cells in spleen and high level of splenic IgA producing cells.

Our newly developed model of orally induced chronic salmonella infection with unique opposite pattern of local and systemic immune reactions in susceptible and resistant mice can be further exploited as a valuable tool in discovery of novel antibacterial therapeutics.

\section{Methods}

2.1. Mice and Infection. Mice of inbred strains I/StSnEgYCit $(\mathrm{I} / \mathrm{St})$ and $\mathrm{A} / \mathrm{JSnYCit}(\mathrm{A} / \mathrm{Sn})$ were maintained under conventional conditions at the Animal Facilities of Gamaleya Research Institute for epidemiology and microbiology, Moscow, Russia, in accordance with national guidelines. Water and food were provided ad libitum. Male mice of 14$16 \mathrm{~g}$ body weight were used in our experiments. S. enterica serovar Typhimurium (var. C53) was grown in broth overnight, centrifuged, resuspended in sterile saline. Serial dilutions were plated on SS-agar for $1.5 \mathrm{~h}$ to estimate colonyforming unit (CFU) numbers, while the bulk sample was stored at $4^{\circ} \mathrm{C}$. The sample was diluted in sterile saline to achieve a concentration of $10^{5} \mathrm{CFU} / \mathrm{mL}$ and $0.2 \mathrm{~mL}(2$ $\times 10^{4} \mathrm{CFU} /$ mouse) was injected per os via gavage needle. Mortality was monitored daily. To estimate salmonella loads, serial dilutions of PP, MLN and spleen homogenates were plated onto SS-agar. Colonies were counted following $12 \mathrm{~h}$ incubation at $37^{\circ} \mathrm{C}$.

2.2. GFP-Labeled Salmonella. To define the intracellular distribution of salmonella in $\mathrm{CD}_{11 \mathrm{c}^{+}}$and $\mathrm{F} 4 / 80^{+}$cells, we performed a separate set of experiments where we used GFPlabeled C53-1 stain of Salmonella, containing GFP-inserted tt115 vector, resistant to kanamycin and ampicillin (kind gift of Professor V. Lunin, laboratory of active nanostructures,
Gamaleya Research Institute, Moscow). In these experiments, spleen cells were stained with R-PE-labeled anti-CD1lc and anti-F4/80 antibodies as described below.

2.3. HङE Histopathology and Immunohistochemistry. Spleens were examined for pathology seven days after infection. Spleen tissue was frozen in the regimen of $-60^{\circ} \mathrm{C}$ to $-20^{\circ} \mathrm{C}$ temperature gradient in the electronic Cryotome (ThermoShandon, Astmoor Runcorn, Cheshire, UK), and serial $8 \mathrm{~mm}$ thick sections were made. Sections were stained with haematoxylin and eosin and further examined for the signs of pathology.

To detect Gr-1-positive cells, sections were preliminary blocked in 1\% BSA for 20 min and stained with FITC conjugated anti-Gr-1 antibodies (clone RB6-8C5, Miltenyi Biotech, Germany) (1/200) or isotype control for $30 \mathrm{~min}$ and washed in PBS 3 times for $10 \mathrm{~min}$. Sections were mounted in Vectashield mounting medium (Vector laboratories, Burlingame, Ca, USA), sealed, examined, and photographed using Nikon Eclipse 50i microscope.

2.4. ELISPOT Assay. Single-cell suspensions were obtained individually from Peyer's patches (PP), mesenteric lymph nodes (MLN), and spleens of mice as described previously [15]. Sterile filter Millipore plates were coated with heat-killed salmonella, washed, and blocked with RPMI-1640 containing $10 \%$ FBS (HyClone, Logan, UT). $2 \times 10^{6}$ cells/wells from three individual animals per group were added to salmonellacoated plate and cultured for $18 \mathrm{~h}$ in $\mathrm{CO}_{2}$ incubator. Then plates were washed and stained with alkaline-phosphataselabeled monoclonal antibodies against mouse IgA, IgG1, and IgG2a (Invitrogen, Carlsbad, CA) washed, and incubated with BCIP/NBT following stop reagent $(0.1 \mathrm{M} \mathrm{HCl})$. Nitrocellulose filters were further scanned with Epson Perfection Photo scanner and analyzed.

2.5. Flow Cytometry. MLN, PP and spleens from I/St and $\mathrm{A} / \mathrm{Sn}$ mice were analyzed at the time points as described below. Lymphoid organs were incubated with collagenase (Invitrogen, Invitrogen, Carlsbad, CA) for 30 minutes on ice, and single-cell suspensions were prepared with the help of Nitex mesh using a syringe plunger. After centrifugation and washing, cells were resuspended in PBS supplemented with $0.5 \% \mathrm{BSA}$ and $0.01 \% \mathrm{NaN}_{3}$ and incubated for $5 \mathrm{~min}$ at $4^{\circ} \mathrm{C}$ with $\mathrm{CD} 16 / \mathrm{CD} 32 \mathrm{mAbs}$ (clone 2.4G2, BD Biosciences, USA) to block Fc-receptors. Cells were then stained with the following directly conjugated antibodies, according to the manufacturer's instructions: R-PE labeled anti-CD1lc or F4/80 (clones N418 and BM8, Invitrogen, Carlsbad, CA), FITC-anti-CD8a (clone 53.67, E-bioscience, San Diego, CA), and FITC-anti-Gr-1 (clone RB6-8C5, Miltenyi Biotech, Germany). Dead cells were excluded with 7-AAD (E-bioscience, San Diego, CA). Two-color staining with either R-PE labeled anti-CD11c and FITC-labeled anti-CD8 antibodies or R-PE labeled anti-F4/80 and FITC-labeled anti-Gr-1 antibodies was performed in our experiments where we did not use GFP-labeled salmonella. In experiments with GFP-labeled salmonella, we used only R-PE labeled anti-CD11c ${ }^{+}$and antiF4/80 antibodies. Stained cells ( $10^{4}$ cells per sample) were 
TABLE 1: Recruitment of CD11c ${ }^{+}$and F4/80 ${ }^{+}$cells into the spleens of mice infected per os with GFP-labeled salmonella.

\begin{tabular}{|c|c|c|c|c|}
\hline & $\%$ of CD11c ${ }^{+}$ & $\%$ of $\mathrm{F} 4 / 80^{+}$ & $\% \mathrm{GFP}^{+} \mathrm{CD} \mathrm{Clc}^{+}$ & $\% \mathrm{GFP}^{+} \mathrm{F} 4 / 80^{+}$ \\
\hline control A/Sn & $0.67 \pm 0.01$ & $2.87 \pm 0.11$ & $0,21 \pm 0.02$ & $0.11 \pm 0.3$ \\
\hline Sal A/Sn & $2.9 \pm 0.1$ & $4.14 \pm 0.01$ & $1.2 \pm 0.25$ & $2.6 \pm 0.1$ \\
\hline control I/St & $2.11 \pm 0.02$ & $0.51 \pm 0.03$ & $0.11 \pm 0.04$ & $0.13 \pm 0.2$ \\
\hline Sal I/St & $14.26 \pm 0.122$ & $5.47 \pm 0.15$ & $12 \pm 1.5$ & $3.38 \pm 0.2$ \\
\hline
\end{tabular}

washed twice and analyzed by flow cytometry, using a FACS Calibur cytometer, CellQuestPro software (BD Biosciences, USA) for acquisition and FCS Express V3 software (De Novo Software, Los Angeles, CA) for analysis. Cells were gated based on their expression of either CD11c and CD8 or Gr1 and F4/80. In experiments with GFP-labeled salmonella gating was performed based on expression of GFP and CD11c or $\mathrm{F} 4 / 80$.

2.6. Statistics. All experiments were performed using at least three samples per group and repeated 3 times. Mean values and standard deviations were calculated using Excel software. The statistical significance was estimated by Student's $t$ test. $P<0.05$ was considered statistically significant.

\section{Results and Discussion}

Recently we have demonstrated that I/St mice display higher susceptibility to acute disease caused by low-dose (0-50 CFU) intraperitoneal salmonella challenge compared to A/Sn mice [14]. Here we report on the course of infection and organ colonization in I/St and A/Sn mice after more natural oral administration of $10^{5}$ CFU S. enterica serovar Typhimurium. In this study, we have found that per os infection with moderately virulent $S$. enterica serovar Typhimurium strain (C53) of both mouse strains resulted in at least 4-week long pathogen persistence in different lymphoid organs (data is not shown). Initially, Peyer's patches, mesenteric lymph nodes, and feces of mice were identically colonized with salmonella, however, between day 5 to 21 after challenge 3 log intrastrain difference in oral $\mathrm{LD}_{50}$ was revealed: $1.0 \times 10^{5} \mathrm{CFU}$ for I/St mice and $5.0 \times 10^{8} \mathrm{CFU}$ for $\mathrm{A} / \mathrm{Sn}$ mice. To the end of these experiments (week 4 after infection) salmonella was found predominantly in MLN in line with earlier observations on pathogen localization in chronic salmonella infection [16]. At day 7 after infection, we have observed the maximum level of splenomegaly in I/St mice (Figure 1). Mean spleen weight of intact I/St control was $41 \mathrm{mg}$ compared to $320 \mathrm{mg}$ in infected mice at day 7 after infection. Mean spleen weight of intact A/Sn controls was $76 \mathrm{mg}$ compared to $190 \mathrm{mg}$ at the same time point that made only 2.5 times increase compared to 8 times spleen increase in I/St mice. Later in the course of infection, these interstrain differences disappeared. The pattern of pathological response in susceptible and resistant mice at day 7 PI correlated with the most pronounced difference in salmonella concentrations in spleens of infected mice $\left(5 \times 10^{6} \mathrm{CFU}-\mathrm{I} / \mathrm{St} ; 2 \times 10^{3} \mathrm{CFU}-\mathrm{A} / \mathrm{Sn}\right)$.

To further analyze interstrain differences in spleen pathology, we performed H\&E staining of spleen sections from I/St and A/Sn mice at day 7 after infection. As shown in

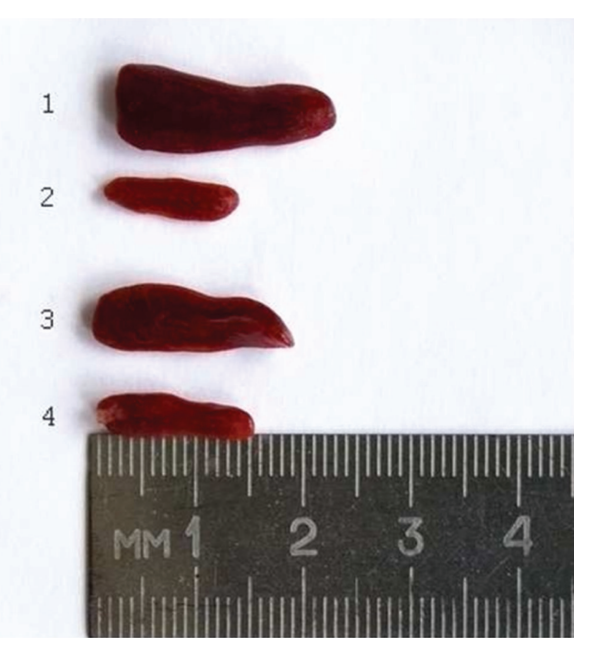

FIGURE 1: Spleens of I/St and A/Sn at day 7 after infection with S. typhimurium and intact controls: 1 Spleen of I/St mouse, day 7 after infection; $2 \mathrm{I} / \mathrm{St}$-intact control. 3 Spleen of A/Sn mouse, day 7 after infection; $4 \mathrm{~A} / \mathrm{Sn}$-intact control.

Figure 2(a), B-cell follicles observed in spleens of both strains had secondary structure at this time point and were more abundant compared to intact controls; however, the increase in B-cell follicles sizes following per os salmonella infection was more pronounced in I/St mice. Cellular infiltration around follicle border was also more pronounced in spleens of I/St mice (Figure 2(b)). To assess cellular involvement in the inflammatory component of the anti-salmonella response, we stained spleen sections with FITC-conjugated anti-Gr-1 antibodies. We found Gr-1-positive cells diffusely distributed around B-cell follicles in controls of both mouse strains (data is not shown). In spleens of infected mice from both mouse strains, we observed dense infiltrates of Gr-1positive cells (Figure 2(c)); however, in spleens of I/St mice, they were more pronounced and hallmarked the sites of necrotic foci. The similar pattern of histopathology with dual B-cell and Gr-1-positive cell involvement was reported in other bacterial models including $M$. avium, M. tuberculosis, L. monocytogenes, and Salmonella [17-19] that might indicate general mechanisms underlying the susceptibility to bacterial infection.

Efficiency of salmonella dissemination to the target organs depends largely on the type of the cells that harbor pathogen [20]. To this end, we have analyzed the distribution of GFP-labeled salmonella in $\mathrm{CD}_{11 c^{+}}$and F4/80 which represent major in vivo cellular targets of infection. Flow cytometry analysis of spleen $\mathrm{CD}_{11 \mathrm{c}^{+}}$and $\mathrm{F} 4 / 80^{+}$cell populations was performed 48 hours after infectious challenge. As shown in the Table 1, I/St had higher level of $\mathrm{GFP}^{+}$ 

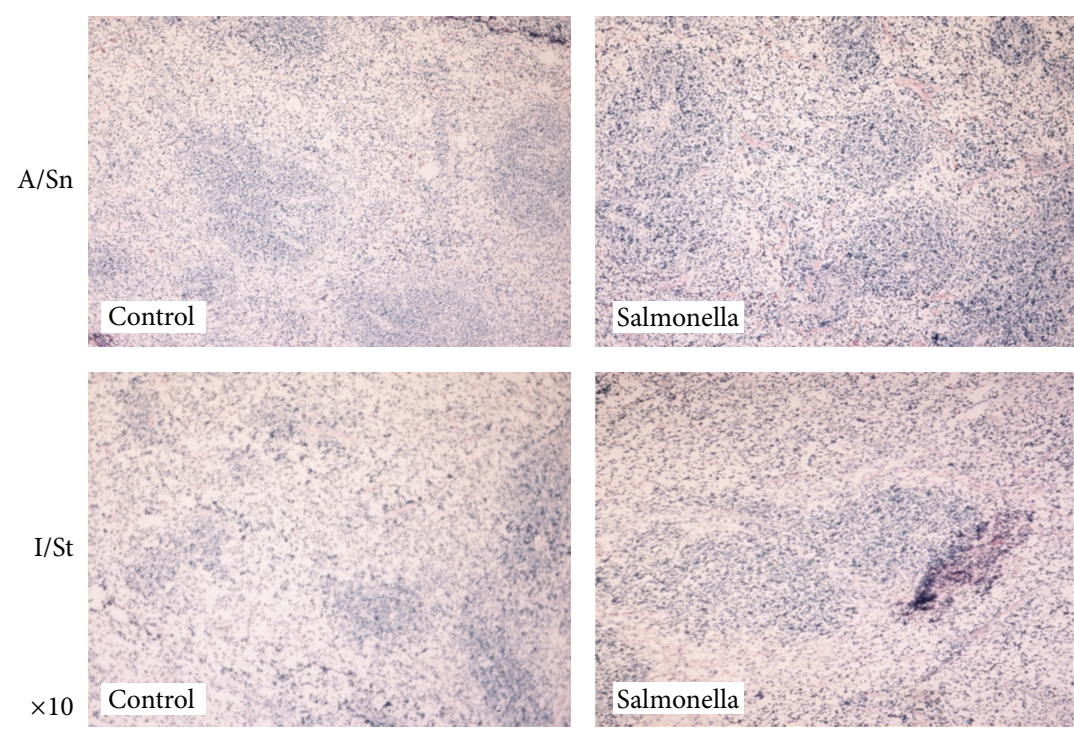

(a) B cell follicles

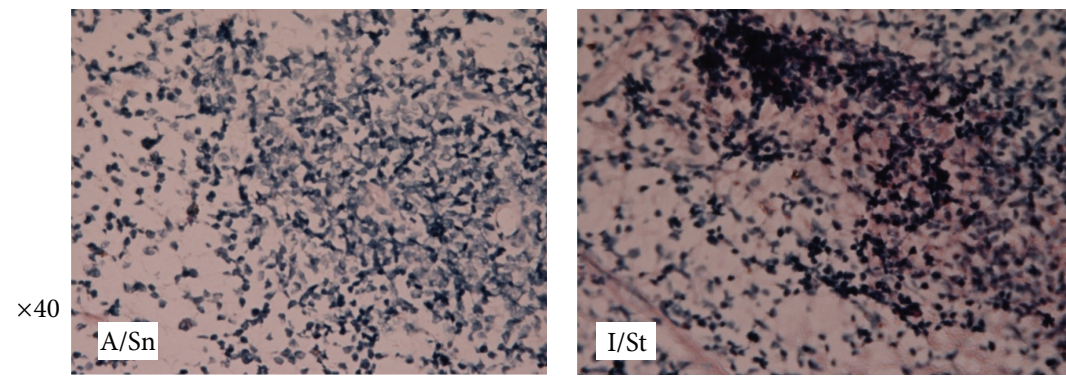

(b) Inflammation foci

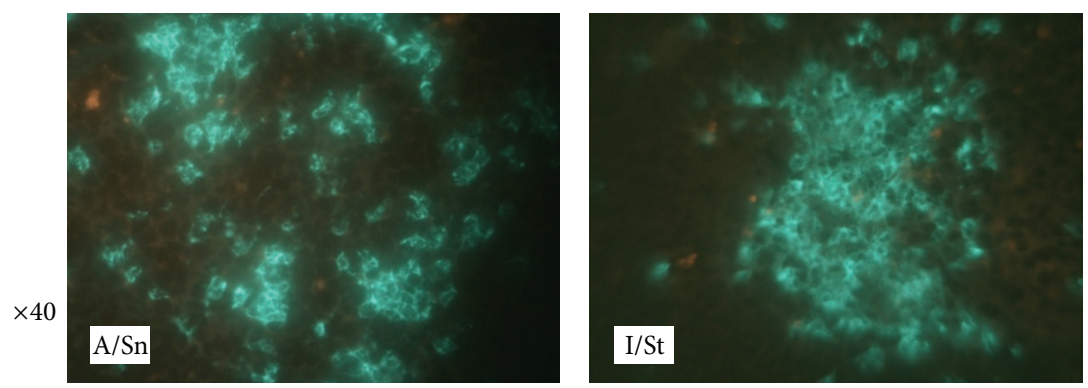

(c) Dense neutrophil infiltrates

Figure 2: (a) H\&E staining of spleens from A/Sn and I/St mice at day 7 after infection, multiplication $\times 10$. (b) Close up of inflammatory elements in spleens of I/St and A/Sn mice, multiplication $\times 40$. (c) Anti-Gr-1 staining in spleens of I/St and I/Sn mice, multiplication $\times 40$.

cells in spleens compared to A/Sn mice. Higher level of GFPlabeled salmonella was observed in $\mathrm{CD}_{11 c^{+}}$cells of I/St mice (12\% in I/St and $1.2 \% \mathrm{~A} / \mathrm{Sn})$, and the number of recruited $\mathrm{CD}_{11 \mathrm{c}^{+}}$cells in spleens of I/St mice was also higher compared to $\mathrm{A} / \mathrm{Sn}$ mice (Table 1 ).

As we observed the greater level of recruitment of CD11c ${ }^{+}$ cells to the spleens of I/St mice (shown in Table 1), we further analyzed intrastrain differences in the cell subset distribution in local lymphoid tissue after oral salmonella challenge. Interestingly, we did not observe any increase in the numbers of "myeloid" $\left(\mathrm{CD} 11 \mathrm{c}^{+}, \mathrm{CD} 8 \mathrm{a}^{-}\right)$dendritic cells in both mouse strains though the level of these cells was higher in PP of intact I/St mice (data is not shown). As shown in the Figure 3, the level of "plasmacytoid" CD11c ${ }^{+} \mathrm{CD} 8 \mathrm{a}^{+}$ dendritic cells was increased in PP of both mouse strains at day 3 after infection, though was higher in I/St mice. No apparent increase in $\mathrm{Grl}^{+}$or $\mathrm{F} 4 / 80^{+}$cells was observed in PP of I/St mice, while the number of these cells was increased in $\mathrm{PP}$ of $\mathrm{A} / \mathrm{Sn}$ mice. The similar pattern of predominantly $\mathrm{CD}_{11 c^{+}}$cell responses was observed in MLN of I/St mice compared predominantly $\mathrm{Gr}-1^{+}$и $\mathrm{F} 4 / 80^{+}$responses seen in resistant $\mathrm{A} / \mathrm{Sn}$ mice. As we did not observe $\mathrm{CD} 11 \mathrm{c}^{+} \mathrm{Gr}-$ $1^{+}$cells either in local lymphoid tissues or in spleens of salmonella-infected A/Sn or I/St mice before (data is not shown), we did not analyze this subpopulation of cells in this study. 


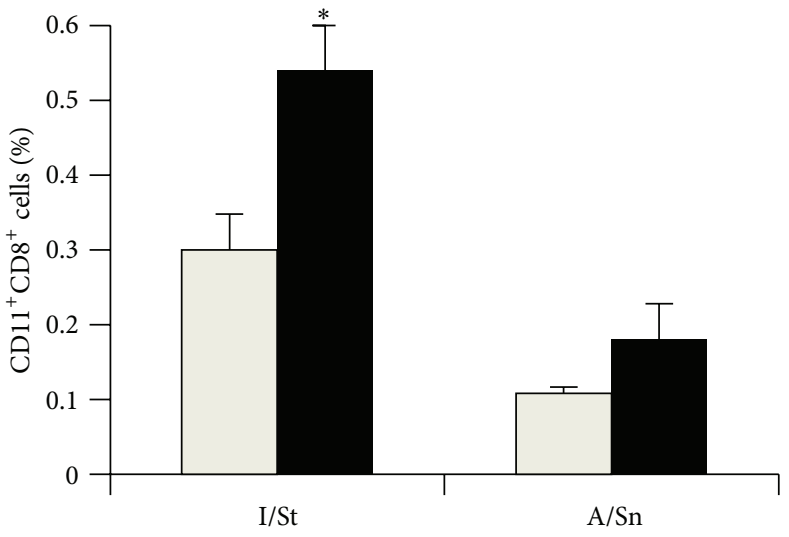

(a)

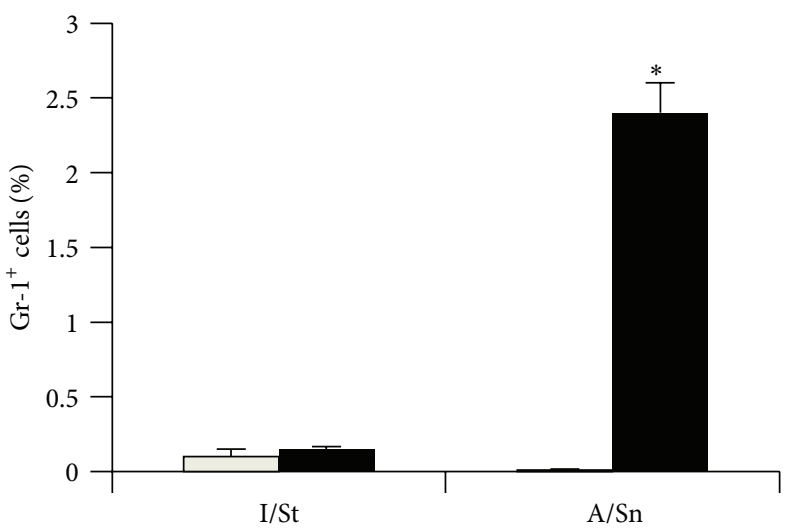

(b)

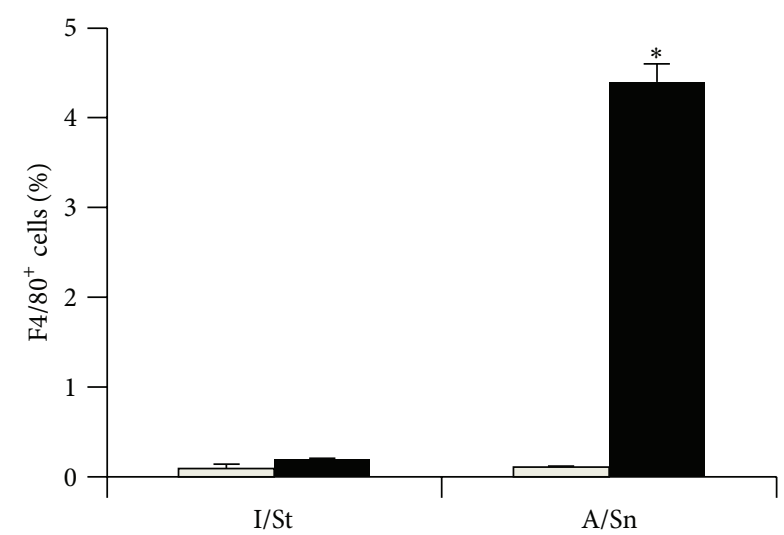

(c)

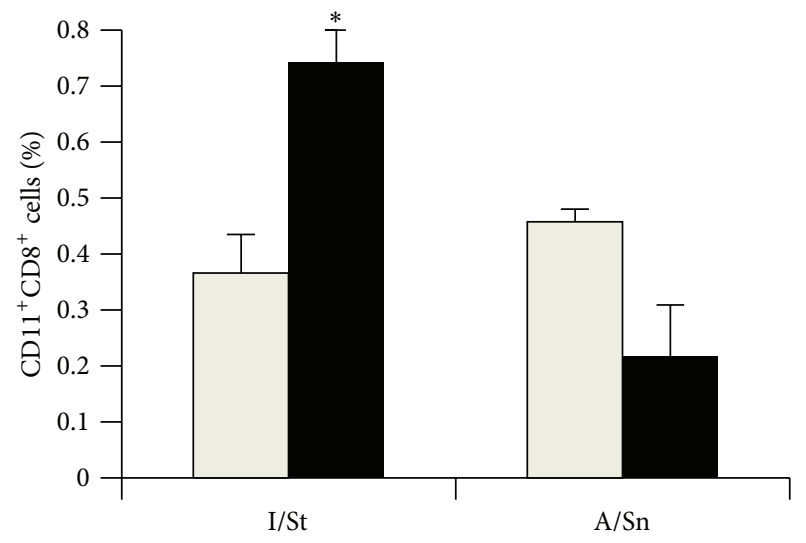

(d)

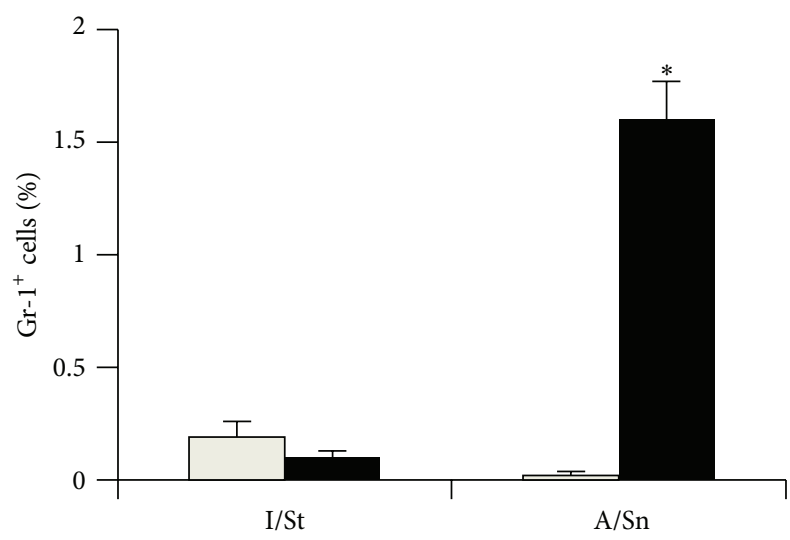

(e)

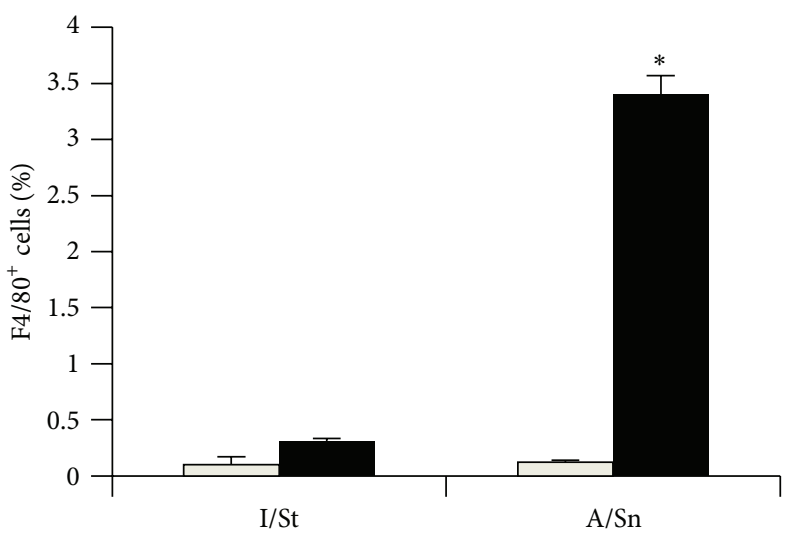

(f)

Figure 3: Distribution of CD11c ${ }^{+} \mathrm{CD} 8 \mathrm{a}^{+}, \mathrm{F} 4 / 80^{+}$, and $\mathrm{Gr}-1^{+}$cells in Peyer's patches and mesenterial lymph nodes at day 3 after oral infection. Light bars: controls, black bars: infected mice. (a)-(c) cell distribution in PP; (d)-(f) cell distribution in MLN. * Statistically significant difference, $P<0.05$.

Thus, early after oral challenge (day 3 after infection), we observed predominant recruitment of $\mathrm{CD}_{11 \mathrm{c}^{+}}$cells to the local lymphoid organs (PP and MLN) in I/St mice, while in $\mathrm{A} / \mathrm{Sn}$ mice $\mathrm{Grl}^{+}$and $\mathrm{F} 4 / 80^{+}$cells were the major local responders with no apparent involvement of $\mathrm{CD}_{11 c^{+}}$cells.

As IgA response is the earliest and the most abundant at mucosal sites and generally assumed as protective, we further studied IgA-producing cells in I/St and $\mathrm{A} / \mathrm{Sn}$ mice both locally (PP) and systemically (spleens) in mice infected per os with $10^{5} \mathrm{CFU}$ of salmonella at days 3,5, and 7 after infection. We also analyzed salmonella-specific IgG2a- and IgG1-producing cells in the same lymphoid organs. As shown in Figure 4, A/Sn mice had higher level of IgA-producing cells in PP compared to I/St at day 3 after infection that probably adds to their antibacterial resistance potential. I/St mice had higher level of IgA-, IgG2a-, and IgG1-producing cells in spleen at day 7 , and this correlated with higher bacterial load and splenomegaly in susceptible mice. 


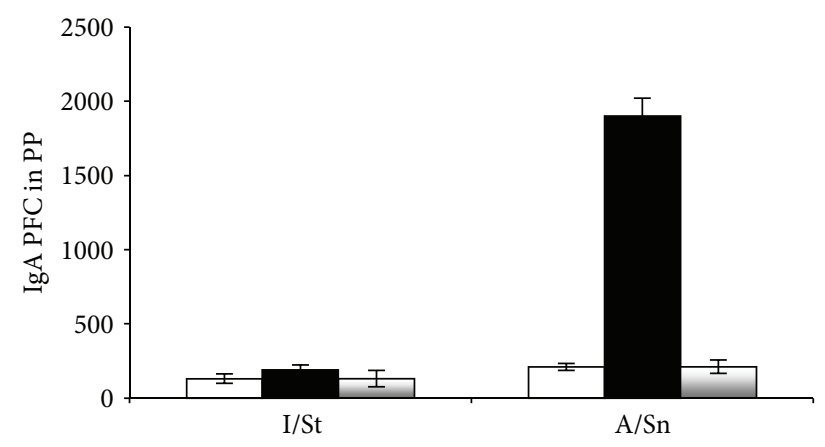

(a)

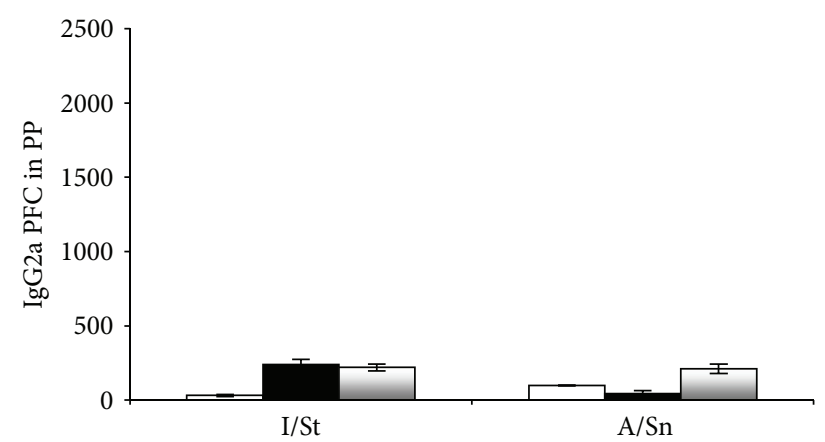

(b)

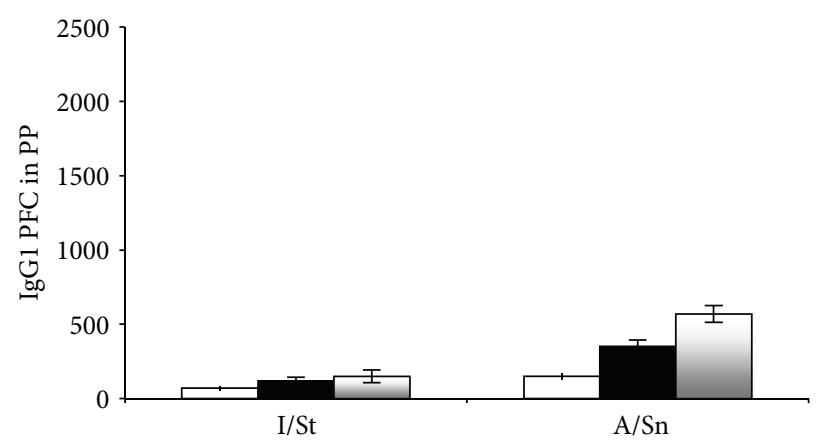

(c)

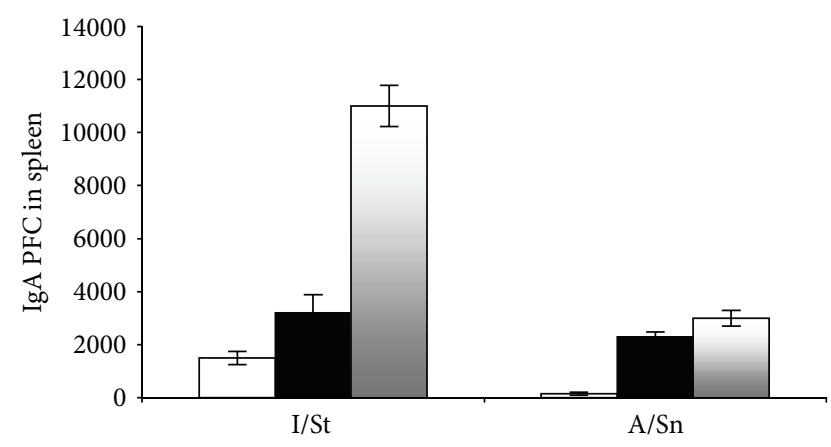

(d)

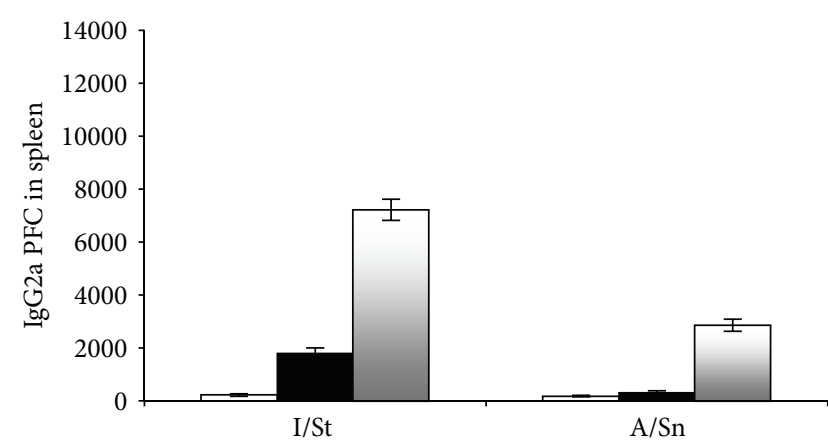

(e)

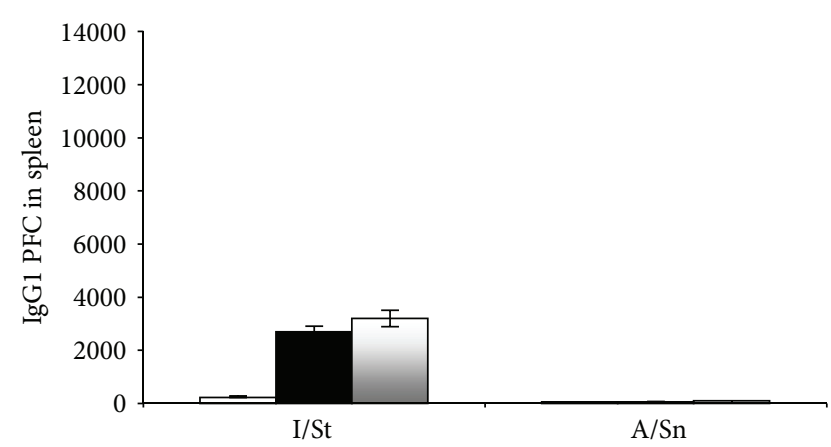

(f)

Figure 4: Numbers of IgA-, IgG2a-, and IgGl-producing cells in PP ((a), (b), and (c)) and spleens ((d), (e), and (f)) of I/St and A/Sn mice. Light bars: day 3, black bars: day 5, grey bars: day 7. * Statistically significant difference, $P<0.05$.

\section{Conclusions}

In this study, we found that susceptible to salmonella I/St mice are defective in their local innate responses to salmonella. They have lower levels of $\mathrm{Gr}-1^{+}$and $\mathrm{F} 4 / 80^{+}$cells in Peyer's patches and mesenterial lymph nodes and reduced numbers of IgA-producing cells in Peyer's patches compared to resistant $\mathrm{A} / \mathrm{Sn}$ mice. In the same time, I/St mice had higher levels of CD11c ${ }^{+}$cells in Peyer's patches and MLN. We also showed that numbers of $\mathrm{CD}_{11 c^{+}}$bearing GFP-labeled salmonella in spleens of I/St mice were higher compared to $\mathrm{A} / \mathrm{Sn}$ mice. Our results suggest that predominantly $\mathrm{CD}_{11 \mathrm{c}^{+}}$ pattern of cell response with lower involvement of $\mathrm{Gr}-\mathrm{I}^{+}$ and $\mathrm{F} 4 / 80^{+}$cells in susceptible mice might contribute to their genetically mediated susceptibility to salmonella. However, direct connection between observed pattern of innate responses and susceptibility in these mice has to be established.

\section{Abbreviations}

PP: Peyer's patches

MLN: Mesenterial lymph nodes

i.p.: Intraperitoneal

CFU: Colony forming unit

$\mathrm{H} \& \mathrm{E}$ : Haematoxylin eosin staining

PFC: Plaque forming cells

PI: $\quad$ Post infection.

\section{Conflict of Interests}

Authors confirm that they have no conflict of interests in the submitted paper.

\section{Acknowledgment}

This work is funded by Russian Basic Research Foundation, project 08-04-01090. 


\section{References}

[1] M. F. Roy and D. Malo, "Genetic regulation of host responses to Salmonella infection in mice," Genes and Immunity, vol. 3, pp. 381-393, 2002.

[2] A. Sansone, P. R. Watson, T. S. Wallis, P. R. Langford, and J. S. Kroll, "The role of two periplasmic copper- and zinc-cofactored superoxide dismutases in the virulence of Salmonella choleraesuis," Microbiology, vol. 148, no. 3, pp. 719-726, 2002.

[3] N. Arpaia, J. Godec, L. Lau et al., "TLR signaling is required for Salmonella typhimurium virulence," Cell, vol. 144, no. 5, pp. 675-688, 2011.

[4] S. S. Pecquet, C. Ehrat, and P. B. Ernst, "Enhancement of mucosal antibody responses to Salmonella typhimurium and the microbial hapten phosphorylcholine in mice with X-linked immunodeficiency by B-cell precursors from the peritoneal cavity," Infection and Immunity, vol. 60, no. 2, pp. 503-509, 1991.

[5] L. N. Nesterenko, N. V. Kobets, and D. V. Balunets, "Model of chronic salmonellosis: parameters of infection and immune response in inbred mice genetically variable in susceptibility to salmonellosis," Zhurnal Mikrobiologii, Epidemiologii, i Immunobiologii, vol. 4, pp. 9-14, 2012.

[6] M. J. Wick, "Innate immune control of Salmonella enterica serovar typhimurium: mechanisms contributing to combating systemic Salmonella infection," Journal of Innate Immunity, vol. 3, no. 6, pp. 543-549, 2011.

[7] L. A. Knodler, B. A. Vallance, J. Celli et al., "Dissemination of invasive Salmonella via bacterial-induced extrusion of mucosal epithelia," Proceedings of the National Academy of Sciences of the United States of America, vol. 107, pp. 17733-17738.

[8] S. E. Winter, M. G. Winter, I. Godinez et al., "A rapid change in virulence gene expression during the transition from the intestinal lumen into tissue promotes systemic dissemination of Salmonella," PLOS Pathogens, vol. 6, no. 8, Article ID e1001060, 2010.

[9] I. Trebichavsky, I. Splíchal, and A. Splíchalová, "Innate immune response in the gut against Salmonella: review," Folia Microbiologica, vol. 55, no. 3, Article ID 20526845, pp. 295-300, 2010.

[10] A. D. Cameron and C. J. Dorman, "fundamental regulatory mechanism operating through OmpR and DNA topology controls expression of Salmonella pathogenicity islands SPI-1 and SPI-2," PLoS Genetics, vol. 8, Article ID e1002615, 2012.

[11] B. Coburn, Y. Li, D. Owen, B. A. Vallance, and B. B. Finlay, "Salmonella enterica serovar Typhimurium pathogenicity island 2 is necessary for complete virulence in a mouse model of infectious enterocolitis," Infection and Immunity, vol. 73, no. 6, pp. 3219-3227, 2005.

[12] J. L. Arques, I. Hautefort, K. Ivory et al., "Salmonella induces flagellin- and MyD88-dependent migration of bacteria-capturing dendritic cells into the gut lumen," Gastroenterology, vol. 137, no. 2, pp. 579-587, 2009.

[13] F. Sánchez, T. V. Radaeva, B. V. Nikonenko et al., "Multigenic control of disease severity after virulent Mycobacterium tuberculosis infection in mice," Infection and Immunity, vol. 71, no. 1, pp. 126-131, 2003.

[14] L. N. Nesterenko, D. V. Balunets, A. S. Tomova et al., "Mycobacterium tuberculosis-susceptible I/St mice develop severe disease following infection with taxonomically distant bacteria, Salmonella enterica and Chlamydia pneumoniae," Clinical and Experimental Immunology, vol. 146, no. 1, pp. 93-100, 2006.

[15] N. Kobets, K. Kennedy, D. O'Donnell, and P. Garside, "An investigation of the ability of orally primed and tolerised $\mathrm{T}$ cells to help B cells upon mucosal challenge," Immunology, vol. 112, no. 4, pp. 550-558, 2004.

[16] D. M. Monack, D. M. Bouley, and S. Falkow, "Salmonella typhimurium persists within macrophages in the mesenteric lymph nodes of chronically infected Nramp1+/+ mice and can be reactivated by IFN $\gamma$ neutralization," Journal of Experimental Medicine, vol. 199, no. 2, pp. 231-241, 2004.

[17] A. A. Navarini, K. S. Lang, A. Verschoor et al., "Innate immuneinduced depletion of bone marrow neutrophils aggravates systemic bacterial infections," Proceedings of the National Academy of Sciences of the United States of America, vol. 106, no. 17, pp. 7107-7112, 2009.

[18] T. K. Kondratieva, E. I. Rubakova, I. A. Linge, V. V. Evstifeev, K. B. Majorov, and A. S. Apt, "B cells delay neutrophil migration toward the site of stimulus: tardiness critical for effective bacillus Calmette-Guérin vaccination against tuberculosis infection in mice," Journal of Immunology, vol. 184, no. 3, pp. 1227-1234, 2010.

[19] E. Kondratieva, N. Logunova, K. Majorov, M. Averbakh, and A. Apt, "Host genetics in granuloma formation: human-like lung pathology in mice with reciprocal genetic susceptibility to $\mathrm{M}$. tuberculosis and M. avium," PloS one, vol. 5, no. 5, p. e10515, 2010.

[20] L. Le Bourhis, J. G. Magalhaes, T. Selvanantham et al., "Role of Nod1 in mucosal dendritic cells during Salmonella pathogenicity island 1-independent Salmonella enterica serovar typhimurium infection," Infection and Immunity, vol. 77, no. 11, pp. 4480-4486, 2009. 


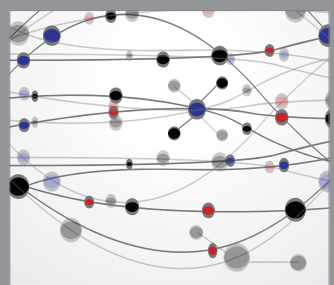

The Scientific World Journal
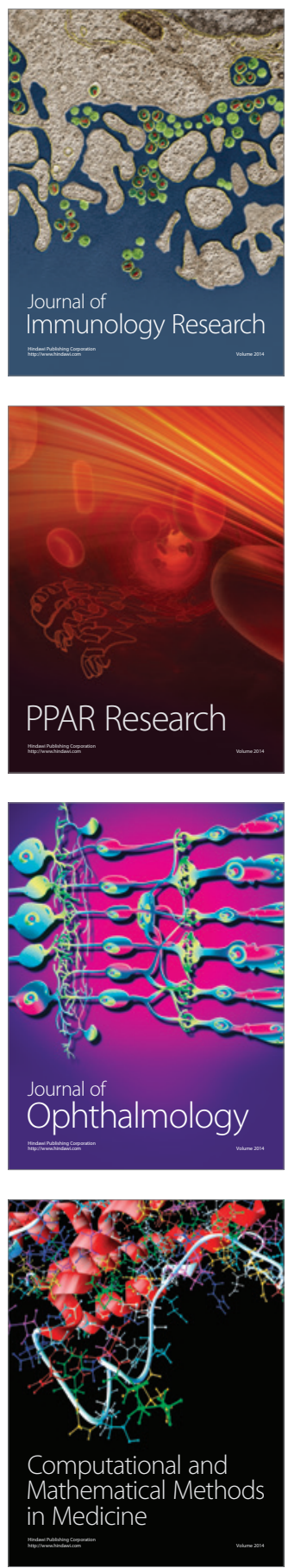

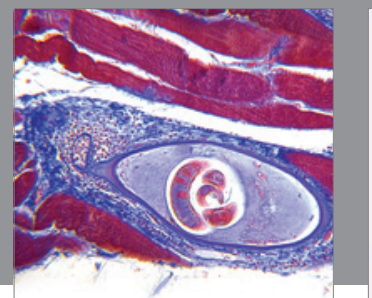

Gastroenterology

Research and Practice
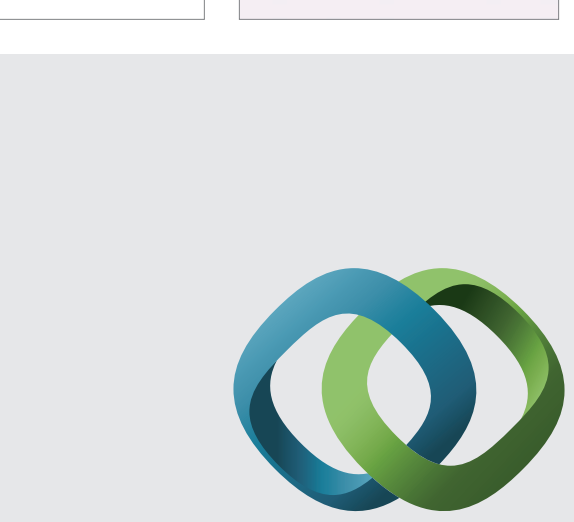

\section{Hindawi}

Submit your manuscripts at

http://www.hindawi.com
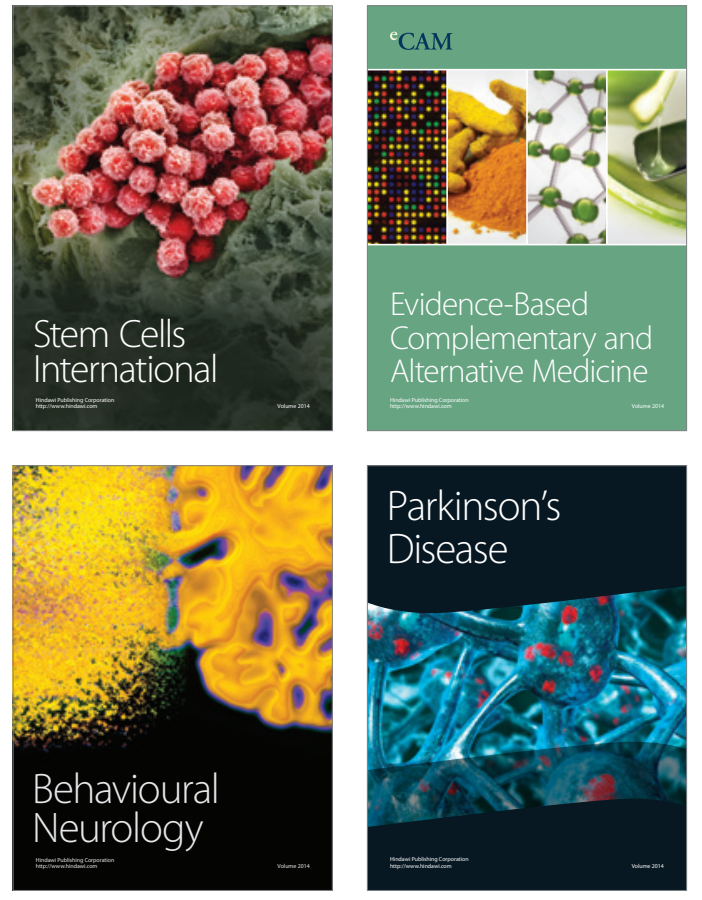
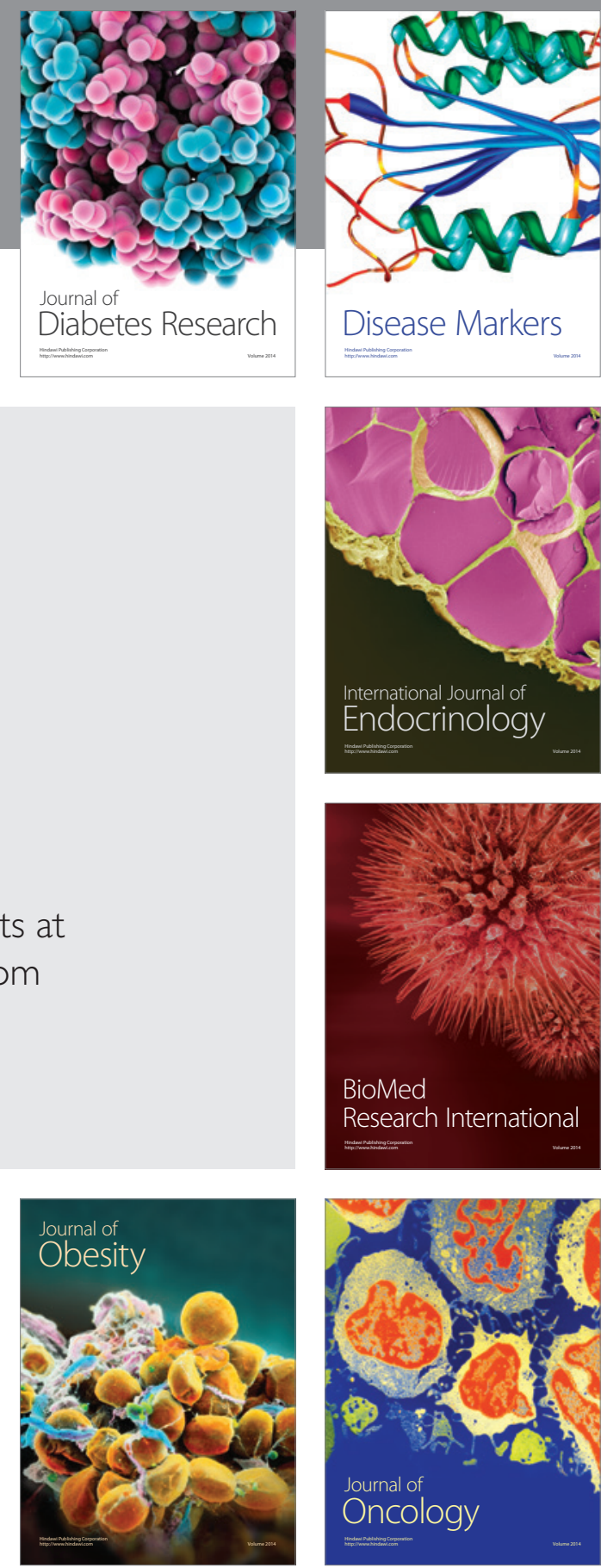

Disease Markers
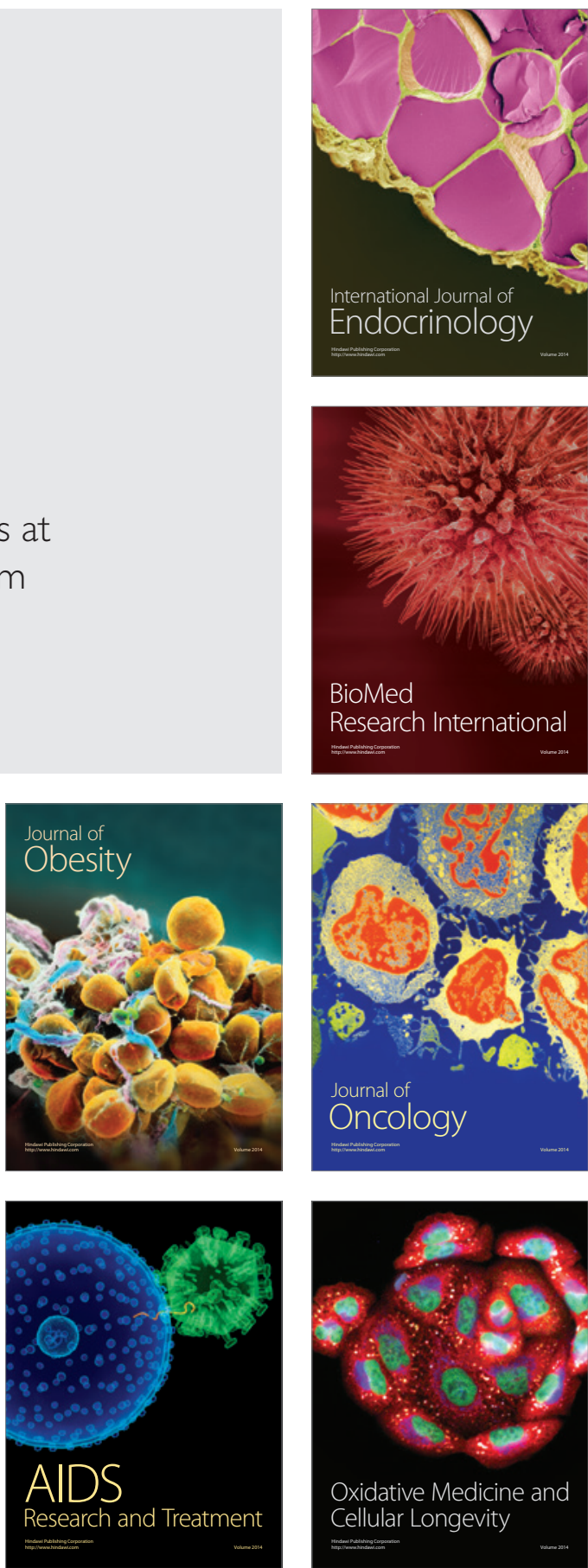